Proceedings

\title{
Comparison of Redox Properties of Flavonoid Aglycones and Corresponding Glycosides and Their Mixtures in the Cellular Model ${ }^{+}$
}

\author{
Zuzanna Koziara 1,*, Monika Baranowska ${ }^{1}$, Agnieszka Bartoszek ${ }^{1}$ and Jacek Namieśnik ${ }^{2}$ \\ 1 Department of Food Chemistry, Technology and Biotechnology, Gdansk University of Technology, \\ 80-233 Gdansk, Poland; monbaran1@student.pg.gda.pl (M.B.); agnieszka.bartoszek@pg.edu.pl (A.B.) \\ 2 Department of Analytical Chemistry, Gdansk University of Technology, 80-233 Gdansk, Poland; \\ jacek.namiesnik@pg.edu.pl \\ * Correspondence: zuzkozia@student.pg.gda.pl \\ + Presented at Natural Products and the Hallmarks of Chronic Diseases-COST Action 16112, \\ Luxemburg, 25-27 March 2019.
}

Published: 18 April 2019

\begin{abstract}
Flavonoids are polyphenolic compounds commonly found in plants. As dietary components, they have been shown to exhibit numerous pro-health properties that are believed to be associated with their antioxidant effects. In this study, the antioxidant activity of four flavonoid compounds was determined by cellular antioxidant activity assay using HT29 cells as a model of the alimentary tract. The strongest impact on cellular redox status was observed for aglycones which acted as both antioxidants (quercetin) and prooxidants (naringenin). Interestingly, mixtures of tested compounds displayed only antioxidative properties.
\end{abstract}

Keywords: cellular antioxidant activity; redox properties; quercetin; rutin; naringin; naringenin

\section{Introduction}

Flavonoids are one of the largest groups of secondary metabolites in the plant kingdom. They are phenolic compounds, which contain in their main structure 15 carbon atoms that form a characteristic carbon skeleton composed of two aromatic rings connected by either tri-carbon bridge or heterocyclic ring. At the highest concentrations, they occur in the epidermis of leaves, flower petals and fruit peel. Therefore, some plant foods are a rich source of these phytochemicals, e.g., fruits, vegetables, tea, coffee, red wine, herbs, spices or chocolate. The results of in vitro and in vivo studies documented the significant impact of flavonoids on redox status of cells. Most data indicate, that antioxidant properties of these compounds result in anti-inflammatory, anticarcinogenic and hepatoprotective properties. However, flavonoids may also act as prooxidants. This prooxidative effect is responsible for the proapoptotic and cytotoxic effects of some compounds belonging to this group.

Flavonoids occur in nature and thus in food products in the form of aglycones or O-glycosides. Very common aglycones are quercetin and naringenin whose glycosides are, respectively, rutin (quercetin-3-rutinoside) and naringin (naringenin-7-rhamnoglucoside). The presence of the sugar residue is known to affect bioactivity, bioavailability and metabolism of flavonoids in the human body [1-3].

The aim of this study was to compare the impact of selected pairs of flavonoids on modulation of cellular antioxidant barrier in the colon adenocarcinoma cell line (HT29) used as a model of alimentary tract to learn how important is the sugar fragment in the structure for their redox properties. 


\section{Materials and Methods}

\subsection{Chemicals, Reagents}

The following compounds were used: quercetin, rutin, naringin and naringenin from SigmaAldrich (Saint Louis, MO), analytical grade ethanol from POCH (Gliwice, Poland). The OxiSelect ${ }^{\mathrm{TM}}$ Cellular Antioxidant Assay Kit was purchased from Cell Biolabs, Inc. (San Diego, CA). All tested compounds were dissolved in $10 \%$ ethanol.

\subsection{Cell Culture}

HT29 cells (human colon adenocarcinoma) from ATCC were used for these studies. Cells were maintained in McCoy's medium with sodium bicarbonate $(2.2 \mathrm{~g} / \mathrm{L})$, L-glutamine $(0.22 \mathrm{~g} / \mathrm{L})$, antibiotics (100 U/mL streptomycin and $100 \mathrm{~g} / \mathrm{L}$ penicillin) and fetal bovine serum $(100 \mathrm{~mL} / \mathrm{L})$. All cell culture chemicals and biochemicals were derived from Sigma-Aldrich (Saint Louis, MO). Cells were kept at $37^{\circ} \mathrm{C}$ under $5 \% \mathrm{CO}_{2}$ atmosphere in a cell incubator (Heal Force, Shanghai, China).

\subsection{CAA (Cellular Antioxidant Activity) Assay}

CAA assay (The OxiSelect ${ }^{\mathrm{TM}}$, Cell Biolabs, Inc., San Diego, CA) was used to assess the cellular antioxidant activity of studied compounds in HT29 cells. The cell suspension was aliquoted $\left(3 \times 10^{4}\right.$ cells per well in $200 \mu \mathrm{L}$ of medium) into 96-well tissue culture black plates with transparent bottoms for fluorescence measurements. The cells were left to settle down for $24 \mathrm{~h}$ at $37^{\circ} \mathrm{C}$. Then, different concentrations of flavonoids were applied (in $50 \mu \mathrm{L}$ ) and the treatment of the cells lasted for $1 \mathrm{~h}$. All treatments were carried out in three technical replicates and three independent experiments were performed. The final concentrations of flavonoids studied covered the range of 1 to $100 \mu \mathrm{M}$. The control cells were treated with $10 \%$ ethanol. Further experimental steps followed the procedure recommended in the manufacturer's website (https://www.cellbiolabs.com). The fluorescence emission was measured at $538 \mathrm{~nm}$ every $5 \mathrm{~min}$ for $1 \mathrm{~h}$ after excitation (at $485 \mathrm{~nm}$ ) using TECAN Infinite M200 plate reader. CAA units were calculated according to the equation:

$$
\text { CAA units }=100-\frac{\mathrm{SA}}{\mathrm{CA}} \times 10
$$

where SA refers to the area under the fluorescence curve, plotted as a function of time, calculated separately for each concentration of tested compounds, while CA is the area under the fluorescence curve as a function of time for control cells treated only with the appropriate solvent [4].

\section{Results}

The CAA assay is a biological test, which is said to effectively mimic the redox conditions found in the human body. It encompasses such aspects as metabolism, bioavailability and distribution of antioxidants in the cell. For this reason, it is believed to better reflect antioxidant activity in vivo than chemical tests. This method relies on the ability of redox-active substances to oxidize or inhibit the oxidation of a fluorescent probe that is absorbed by cells. The intensity of fluorescence is directly proportional to the degree of oxidation of the probe, which allows to assess the anti- or pro-oxidative capacity of the test substance in a cell model used. The results of fluorescence measurements are presented as CAA units. Higher values of CAA units indicate stronger antioxidant properties, while negative values suggest prooxidative activity $[4,5]$.

In this study, CAA assay was performed for four flavonoid compounds which constituted pairs aglycone-glycoside. These were quercetin and its rutinoside-rutin and naringenin and its rhamnoglucoside-naringin. The results obtained revealed that naringin negligibly influenced cellular redox properties within the concentration range studied (Figure 1). All remaining compounds affected cellular redox status in a dose dependent manner but differed substantially in their effects: quercetin was the strongest antioxidant, rutin displayed weak antioxidant properties at 
the highest concentration tested, while CAA units for naringenin decreased with increasing concentration reaching negative values, which is indicative of prooxidative properties.
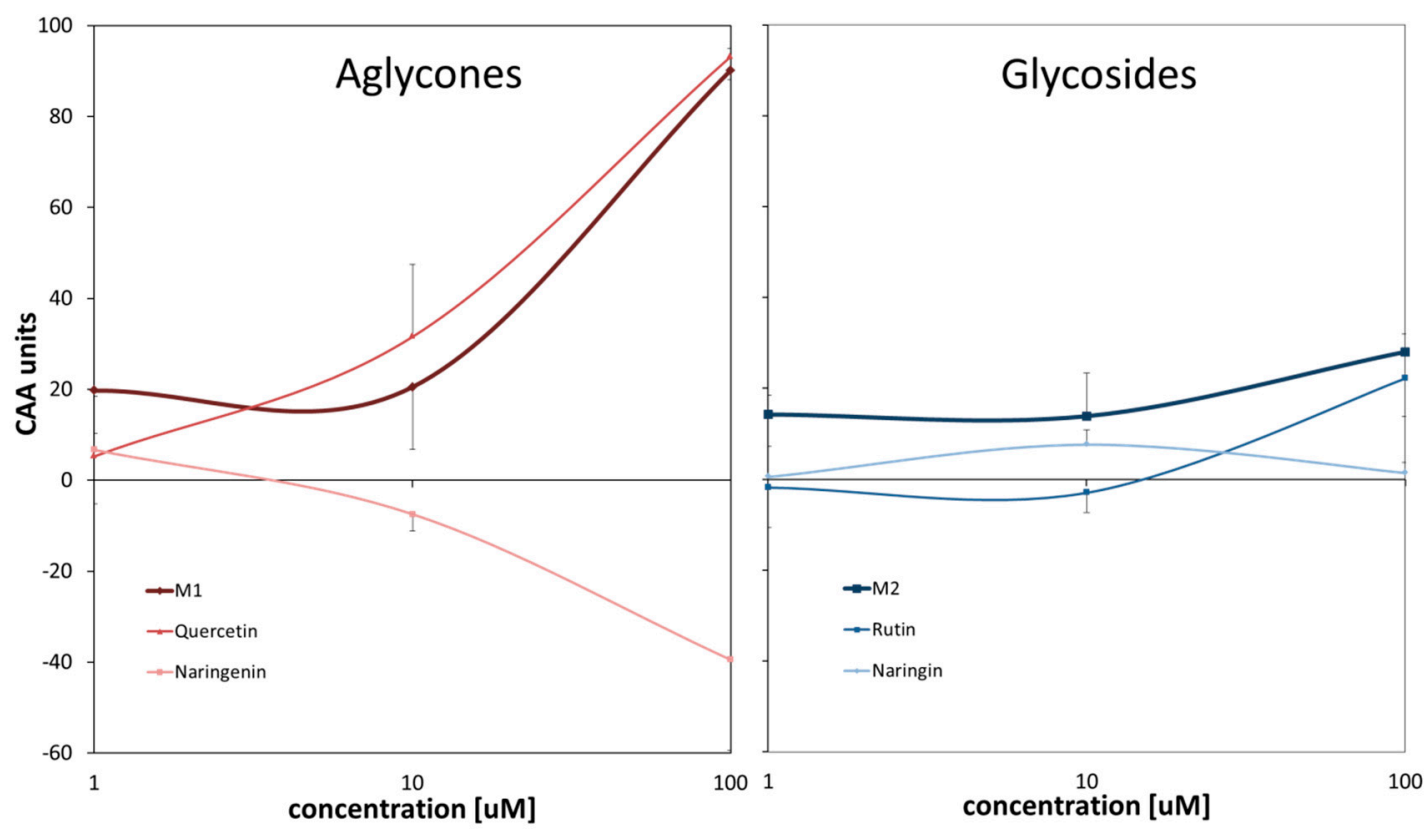

Figure 1. The relationship between cellular antioxidant activity (expressed as CAA units) and the concentration of rutin, quercetin, naringin, naringenin and their mixtures. The results are means of three independent experiments carried out in triplicates.

In the next series of experiments, the cellular redox activity of two mixtures of compounds studied was examined (Figure 1). The mixture M1 contained aglycones (quercetin and naringenin); the mixture M2 included glycosides (rutin and naringin). In contrary to expectations, such a combined treatment of HT29 cells did not result in averaged effects and CAA values determined tended to reflect only antioxidant activity of mixtures' components.

\section{Discussion}

This study determined the effect of selected flavonoids, aglycones and corresponding glycosides, and their mixtures on cellular antioxidant activity in the colon adenocarcinoma cell line (HT29) model. In general, it was observed that aglycones are stronger modulators of cellular redox homeostasis. The results obtained for rutin and quercetin revealed a dose dependent antioxidant activity of these compounds. Their physiological concentrations $(1-10 \mu \mathrm{M})$ did not significantly affect the antioxidant status of cells. However, higher concentration $(100 \mu \mathrm{M})$, which may reflect intestinal epithelium cells being in contact with food ingested, strongly enhanced cellular antioxidant protection, especially in the case of quercetin. For naringin, differences in CAA units for all tested concentrations were small and oscillated around zero, while naringenin exhibited prooxidative properties at higher concentrations tested. Therefore, consumption of these two flavonoids in a purified form, e.g., isolated from citrus fruits, may not offer expected protection against oxidative stress. The presence of sugar residue seemed to quench these strong reducing or oxidative responses leading to the maintenance of proper cellular redox homeostasis. Alternatively, the lower activity of glycosides may result from their more hydrophilic nature, which decreases their bioavailability, thus negatively affecting concentration inside cells.

Interestingly, neither of mixtures showed cellular antioxidant activity that would indicate the additive effect of two components. The presence of the substance with prooxidant activity (naringenin) did not diminish the impact of the antioxidant (quercetin). The response of cells to the treatment with the combination of flavonoids seemed to be only affected by their antioxidant properties, while the prooxidant ones did not influence cellular redox status. This observation 
suggests that the cellular redox system, when supported by exogenous antioxidants may successfully resist the exposure to prooxidants.

Author Contributions: Conceptualization, A.B.; methodology, M.B.; investigation, Z.K. and M.B.; writingoriginal draft preparation, Z.K.; writing - review and editing, A.B., funding acquisition, J.N.

Funding: Project "Antioxidant Power Series as a tool rational design and assessment of health promoting properties of functional food based on antioxidant phytochemicals" (number of the application 2014/14/A/ST4/00640) financed by National Science Centre, Poland in a programme "MAESTRO 6".

Acknowledgments: This article is based upon work from COST Action NutRedOx-CA16112 supported by COST (European Cooperation in Science and Technology).

Conflicts of Interest: The authors declare no conflict of interest.

\section{References}

1. Heim, K.E.; Tagliaferro, A.R.; Bobilya D.J. Flavonoid antioxidants: Chemistry, metabolism and structureactivity relationships. J. Nutr. Biochem. 2002, 13, 572-584., doi:10.1016/S0955-2863(02)00208-5.

2. Kumar, S.; Pandey, A.K. Chemistry and biological activities of flavonoids: An overview. Sci. World J., 2013, Article ID 162750, 16 pages, doi:10.1155/2013/162750.

3. Crozier, A.; Jaganath, I.B.; Clifford, M.N. Phenols, polyphenols and tannins: An overview. In Plant Secondary Metabolites; 1st ed.; Crozier, A., Clifford, M.N., Ashihara, H., Eds.; Blackwell Publishing Ltd: Hoboken, NJ, USA, 2006; pp. 1-22, doi:10.1002/9780470988558.

4. Baranowska, M.; Suliborska, K.; Chrzanowski, W.; Kusznierewicz, B.; Namieśnik, J.; Bartoszek, A. The relationship between standard reduction potentials of catechins and biological activities involved in redox control. Redox Biol. 2018, 17, 355-366, doi:10.1016/j.redox.2018.05.005.

5. Wolfe, K.L.; Liu, R.H. Cellular antioxidant activity (CAA) assay for assessing antioxidants, foods, and dietary supplements. J. Agric. Food Chem. 2007, 55, 8896-8907, doi:10.1021/jf0715166.

(C) 2019 by the authors. Licensee MDPI, Basel, Switzerland. This article is an open access article distributed under the terms and conditions of the Creative Commons Attribution (CC BY) license (http://creativecommons.org/licenses/by/4.0/). 